\title{
An Ontological Approach to Oracle BPM
}

\author{
Jean Prater, Ralf Mueller, Bill Beauregard \\ Oracle Corporation, 500 Oracle Parkway, Redwood City, CA 94065, USA \\ jean.prater@oracle.com, ralf.mueller@oracle.com, william.beauregard@oracle.com
}

\begin{abstract}
The Oracle Business Process Management (Oracle BPM) Suite is composed of tools for Business Analysts and Developers for the modeling of Business Processes in BPMN 2.0 (OMG ${ }^{1}$ standard), Business Rules, Human Workflow, Complex Events, and many other tools. BPM operates using the common tenants of an underlying Service Oriented Architecture (SOA) runtime infrastructure based on the Service Component Architecture (SCA). Oracle Database Semantic Technologies provides native storage, querying and inferencing that are compliant with W3C standards for semantic (RDF/OWL) data and ontologies, with scalability and security for enterprise-scale semantic applications.

Semantically-enabling all artifacts of BPM from the high-level design of a Business Process Diagram to the deployment and runtime model of a BPM application promotes continuous process refinement, enables comprehensive impact analysis and prevents unnecessary proliferation of processes and services. This paper presents the Oracle BPM ontology based upon BPMN 2.0, Service Component Architecture (SCA) and the Web Ontology Language (OWL 2). The implementation of this ontology provides a wide range of use cases in the areas of Process Analysis, Governance, Business Intelligence and Systems Management. It also has the potential to bring together stakeholders across an Enterprise, for a true Agile End-to-End Enterprise Architecture. Example use cases are presented as well as an outlook of the evolution of the ontology to cover the organizational and social aspects of Business Process Management.
\end{abstract}

\section{Introduction}

In the 1968 film, 2001: A Space Odyssey, the movie's antagonist, HAL, is a computer that is capable not only of speech, speech recognition, and natural language processing, but also lip reading, apparent art appreciation, interpreting and reproducing emotional behavior, reasoning, and playing chess, all while maintaining the systems on an interplanetary mission. While the solution we present in this paper does not possess all of the capabilities of HAL, the potential benefits of combining semantic technology with Oracle BPM provides the ability to define contextual relationships between business processes and provides the tools to use that context so that 'software agents' (programs working on behalf of people) can find the right

${ }^{1}$ Object Management Group, see http://www.omg.org 
information or processes and make decisions based on the established contextual relationships.

Organizations can more efficiently and effectively optimize their information technology resources through a service-oriented approach leveraging common business processes and semantics throughout their enterprise. The challenge, however, with applications built on Business Process Management (BPM) and Service Oriented Architecture (SOA) technology is that many are comprised of numerous artifacts spanning a wide range of representation formats. BPMN 2.0, the Service Component Architecture Assembly Model, Web Service definitions (in the form of WSDL), XSLT transformations, for example are all based on well defined but varying type models. To answer even simple queries on the entire BPM model, a user is left with a multitude of API's and technologies, making the exercise difficult and highly complicated. Oracle has developed an ontology in OWL that encompasses all the artifacts of a BPM application and is stored in Oracle Database Semantic Technologies that provides a holistic view of the entire model and a unified and standardized way to query that model using SPARQL.

Oracle is actively involved in the standards process and is leading industry efforts to use ontologies for metadata analysis. Oracle is also investigating the integration of organizational and social aspects of BPM using $\mathrm{FOAF}^{2}$. BPMN 2.0 task performers can be associated with a FOAF Person, Group or Organization and then used in Social Web activities to enable Business Users to collaborate on BPM models.

\subsection{Benefits}

The benefits of adding semantic technology to the database and to business process management in the middleware, driven by an underlying ontology are three fold:

1. It promotes continuous process refinement. A less comprehensive process model can evolve into a complete executable process in the same model.

2. It makes it easy to analyze the impact of adding, modifying or deleting processes and process building blocks on existing processes and web services.

3. It helps prevent unnecessary proliferation of processes and services.

Combining semantic technology and business process management allows business users across organizational boundaries to find, share, and combine information and processes more easily by adding contextual relationships.

\subsection{Customer Use Case}

The US Department of Defense (DoD) is leading the way in the Federal Government for Architecture-driven Business Operations Transformation. A vital tenet for success is ensuring that business process models are based on a standardized representation, thus enabling the analysis and comparison of end to end business processes. This will lead to the reuse of the most efficient and effective process patterns (style guide), comprised of elements (primitives), throughout the DoD Business Mission Area. A key principle in DoD Business Transformation is its focus on data ontology. The

\footnotetext{
${ }^{2}$ The Friend of a Friend (FOAF) project, see http://www.foaf-project.org
} 
Business Transformation Agency (BTA), under the purview of the Deputy Chief Management Officer (DCMO), has been at the forefront of efforts to develop a common vocabulary and processes in support of business enterprise interoperability through data standardization. The use of primitives and reuse of process patterns will reduce waste in overhead costs, process duplication and building and maintaining enterprise architectures. By aligning the Department of Defense Architecture Framework $^{3} 2.0$ (DoDAF 2.0) with Business Process Modeling Notation 2.0 (BPMN 2.0) and partnering with industry, the BTA is accelerating the adoption of these standards to improve government business process efficiency.

\section{The Oracle BPM Ontology}

The Oracle BPM ontology encompasses and expands the BPMN 2.0 and SCA ontologies. The Oracle BPM ontology is stored in Oracle Database Semantic Technologies and creates a composite model by establishing relationships between the OWL classes of the BPMN 2.0 ontology and the OWL classes of the SCA runtime ontology. For example, the BPMN 2.0 Process, User Task and Business Rule Task are mapped to components in the composite model. Send, Receive and Service Tasks, as well as Message Events are mapped to appropriate SCA Services and References and appropriate connections are created between the composite model artifacts. Figure 1 illustrates the anatomy of the Business Rule Task "Determine Approval Flow" that is a part of a Sales Quote demo delivered with BPM Suite.

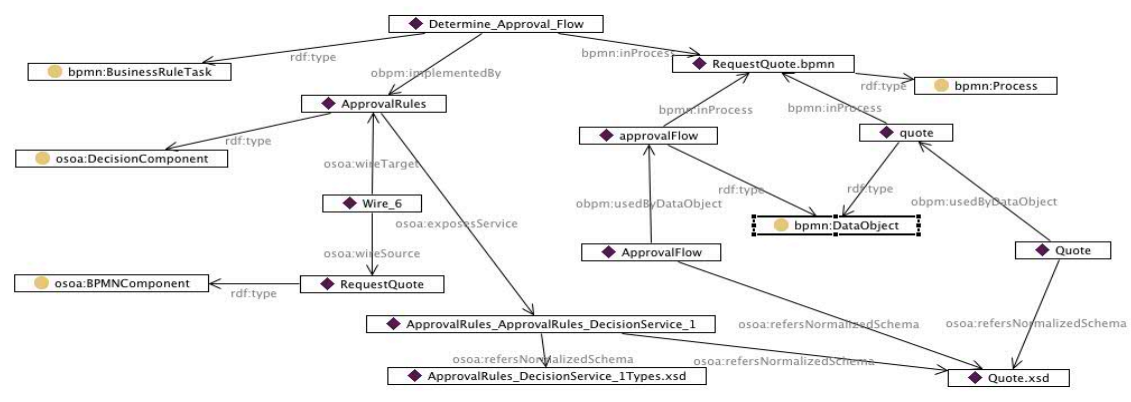

Figure 1: Anatomy of a BPMN 2.0 Business Rule Task ${ }^{4}$

The diagram shows that the Business Rule Task "Determine Approval Flow" is of BPMN 2.0 type Business Rule Task and implemented by a SCA Decision Component that is connected to a BPMN Component "RequestQuote". Also of significance is that the Decision Component exposes a Service that refers to a specific XML-Schema, which is also referred to by Data Objects in the BPMN 2.0 process RequestQuote.bpmn.

${ }^{3}$ See http://www.bta.mil/products/BEA_6.2/BEA/products/2009-04-27 Primitives Guidelines for Business Process Models (DoDAF OV-6c).pdf

${ }^{4}$ Visualized using TopBraid Composer ${ }^{\mathrm{TM}}$ 


\section{An Ontology for BPMN 2.0}

With the release of the OMG BPMN 2.0 standard, a format based on XMI and XML-Schema was introduced for the Diagram Interchange (DI) and the Semantic Model. Based on the BPMN 2.0 Semantic Model, Oracle created an ontology that is comprised of the following:

- OWL classes and properties for all BPMN 2.0 Elements that are relevant for the Business Process Model. ${ }^{6}$ The OWL classes, whenever possible, follow the conventions in the BPMN 2.0 UML meta model. OWL properties and restrictions are included by adding all of the data and object properties according to the attributes and class associations in the BPMN 2.0 model. ${ }^{7}$

- OWL classes and properties for instantiations of a BPMN 2.0 process model. These OWL classes cover the runtime aspects of a BPMN 2.0 process when executed by a process engine. The process engine creates BPMN 2.0 flow element instances when the process is executed. Activity logging information is captured, including timestamps for a flow element instance's activation and completion, as well as the performer of the task.

The implicit (unstated) relationships in the Oracle BPM ontology can be automatically discovered using the native inferencing engine included with Oracle Database Semantic Technologies. The explicit and implicit relationships in the ontology can be queried using Oracle Database Semantic Technologies support for SPARQL (patterns matching queries) and/or mixed SPARQL in SQL queries. [6] Example SPARQL queries are shown below:

Select all User Tasks in all Lanes

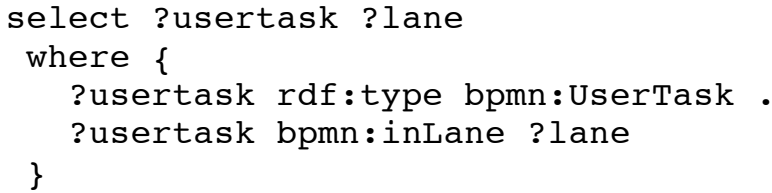

Select all flow elements with their sequence flow in lane p1:MyLane (a concrete instance of RDF type bpmn:Lane)

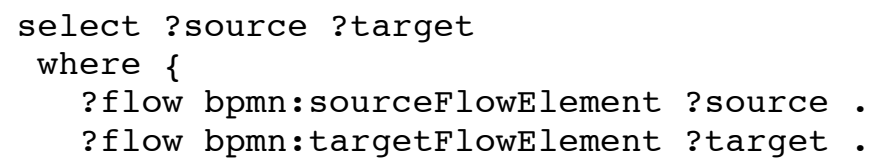

${ }^{5}$ All of the classes of the BPMN 2.0 meta model that exists for technical reasons only (model $\mathrm{m}: \mathrm{n}$ relationship or special containments) are not represented in the ontology

${ }^{6}$ The work in [2] describes an ontology based on BPMN 1.x for which no standardized meta model exists

${ }^{7}$ Oracle formulated SPARQL queries for envisioned use cases and added additional properties and restrictions to the ontology to support those use cases 
\}

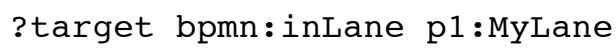

Select all activities in process p1:MyProcess that satisfy SLA p1:MySLA

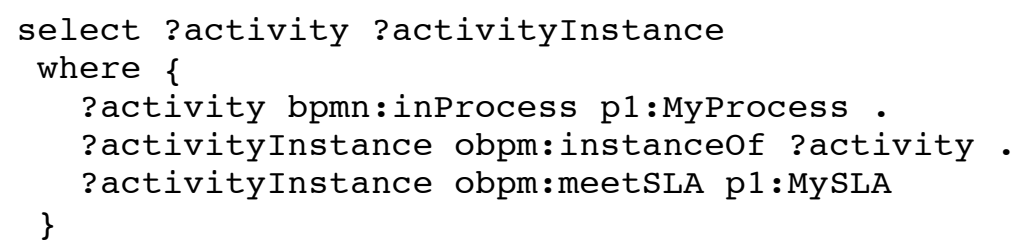

A unique capability of BPMN 2.0, as compared to BPEL, for instance, is its ability to promote continuous process refinement. A less comprehensive process model, perhaps created by a business analyst can evolve into a complete executable process that can be implemented by IT in the same model. The work sited in Validating Process Refinement with Ontologies[4] suggests an ontological approach for the validation of such process refinements.

\section{An Ontology for the SCA composite model}

The SCA composite model ontology represents the SCA assembly model and is comprised of OWL classes for Composite, Component, Service, Reference and Wire, which form the major building blocks of the assembly model. Oracle BPM ontology has OWL classes for concrete services specified by WSDL and data structures specified by XML-Schema. The transformation of the SCA assembly model to the SCA ontology includes creating finer grained WSDL and XML-Schema artifacts to capture the dependencies and relationships between concrete WSDL operations and messages to elements of some XML-Schema and their imported schemata.

The SCA ontology was primarily created for the purpose of Governance and to act as a bridge between the Oracle BPM ontology and an ontology that would represent a concrete runtime infrastructure. This enables the important ability to perform impact analysis to determine, for instance, which BPMN 2.0 data objects and/or data associations are impacted by the modification of an XML-Schema element or which Web Service depends on this element. This feature helps prevent the proliferation of new types and services, and allows IT to ascertain the impact of an XML-Schema modification.

\section{The Technologies}

As part of the customer use case, as referenced in section 1.2 above, we implemented a system that takes a BPM Project comprised of BPMN 2.0 process definitions, SCA assembly model, WSDL service definitions, XML-Schema and other metadata, and created appropriate Semantic data (RDF triples) for the Oracle BPM ontology. The 
triples were then loaded into Oracle Database Semantic Technologies [3] and a SPARQL endpoint was used to except and process queries.

\section{Conclusion}

Oracle BPM ontology encompasses and expands the generic ontologies for BPMN 2.0 and the SOA composite model to cover all artifacts of a BPM application from a potentially underspecified ${ }^{8}$ process model in BPMN 2.0 down to the XML-Schema element and type level at runtime for process analysis, governance and Business Intelligence. The combination of RDF/OWL data storage, inferencing and SPARQL querying, as supported by Oracle Database Semantic Technologies, provides the ability to discover implicit relationships in data and find implicit and explicit relationships with pattern matching queries that go beyond classical approaches of XML-Schema, XQuery and SQL.

\section{Acknowledgements}

We'd like to thank Sudeer Bhoja, Linus Chow, Xavier Lopez, Bhagat Nainani and Zhe Wu for their contributions to the paper and valuable comments.

\section{References}

[1] Business Process Model and Notation (BPMN) Version 2.0, http://www.omg.org/spec/BPMN/2.0/

[2] Ghidini Ch., Rospocher M., Serafini L.: BPMN Ontology, https://dkm.fbk.eu/index.php/BPMN_Ontology

[3] Oracle Database Semantic Technologies, http://www.oracle.com/technetwork/database/options/semantic-tech/

[4] Ren Y., Groener G., Lemcke J., Tirdad R., Friesen A., Yuting Z., Pan J., Staab S.: Validating Process Refinement with Ontologies

[5] Service Component Architecture (SCA), http://www.osoa.org

[6] Kolovski V., Wu Z., Eadon G.: Optimizing Enterprise-Scale OWL 2 RL Reasoning in a Relational Database System, ISWC 2010, page 436-452

[7] "Use of End-toEnd (E2E) Business Models and Ontology in DoD Business Architectures"; Memorandum from Deputy Chief Management Office; April 4, 2011, Elizabeth A. McGrath, Deputy DCMO.

[8] "Primitives and Style: A Common Vocabulary for BPM across the Enterprise"; Dennis Wisnosky, Chief Architect \& CTO ODCMO and Linus Chow Oracle; BPM Excellence in Practice 2010; Published by Future Strategies, 2010

${ }^{8}$ A BPMN 2.0 model element is considered underspecified, if its valid but not all attribute values relevant for execution are specified. 